\title{
"Significados de las Relaciones de Pareja en las y los Estudiantes Universitarios/as y sus Implicancias en sus Interacciones Sociales de la Universidad Nacional Jorge Basadre Grohmann Tacna, 2011"
}

\section{"Significances on Jorge Basadre Grohoman National University Couples of Students and the Implications in their Social Interactions Tacna, 2011"}

\author{
Carmen Luisa Linares Torres, ${ }^{2}$ Gema Natividad Sologuren García.
}

\begin{abstract}
RESUMEN:
Es una investigación cualitativa, responde a la pregunta: ¿Cuáles son los significados que tienen los/las estudiantes universitarios/as de la UNJBG sobre sus relaciones de pareja y las implicancias que estos significados tienen en sus interacciones sociales? Entre los significados que asignan a la relación de pareja están el reconocimiento de la identidad y la necesidad de compartir sentimientos y espacios de la vida personal con otra persona. Ellos/as basan sus relaciones en las interacciones de uno y otro miembro de la pareja; en sus interacciones sociales están dispuestos a compartir sentimientos, mostrar conductas y actitudes para fortalecer el vínculo amoroso y excluir a los demás de su relación. Se observa el temor de los hombres al fracaso matrimonial: proponen una fase de convivencia para estar seguros. Este temor no parece estar presente en las mujeres que proyectan su relación hacia el matrimonio como parte de suimaginario.
\end{abstract}

Palabras clave: relaciones de pareja, interacciones sociales.

\section{ABSTRACT:}

It is a qualitative research which answers the following question: What significances do UNJBG university students have in their relationships and as a result of them what the implications are in their social interactions? Among the significances they attach to the relationship is the recognition of the identity and the need of sharing feelings and areas of their personal life with another person. They base their relationships in interactions of each other; in their social interactions they are willing to share feelings, behaviors and show attitudes to strengthen bonds of love and exclude others from their relationship. It is clear that the fear of men to marriage failure: they propose a phase of cohabitation to be sure. This fear does not seem to be present in women who project their relationship towards marriage as part of their way of thinking.

Keywords: relationships, social interactions.

\footnotetext{
Magister en Docencia Universitaria, Especialista en Salud Reproductiva de los y las Adolescentes, Licenciada en Obstetricia. Facultad de Ciencias de la Salud. Universidad Nacional Jorge Basadre Grohmann.

${ }^{2}$ Magister en Docencia Universitaria, Especialista en Salud Reproductiva de los y las Adolescentes, Licenciada en Obstetricia. Facultad de Ciencias de la Salud. Universidad Nacional Jorge Basadre Grohmann.
} 


\section{INTRODUCCIÓN}

El ser humano es un ser social y como tal interactúa con otras personas estableciendo diferentes tipos de relaciones interpersonales. Entre los tipos más importantes se encuentran las relaciones con los amigos, la familia y la pareja (noviazgo). Sin embargo, las de pareja son la que traen consigo más conflictos, tensiones y desilusiones, que otro tipo de relación. Garduño; 1999, 26. En la investigación que se presenta se trata de responder a la siguiente interrogante:

¿Cuáles son los significados que dan los/las estudiantes universitarios/as sobre sus relaciones de pareja y las implicancias que estos significados tienen en sus interacciones sociales?

El objetivo general es analizar los significados que dan los estudiantes universitarios sobre sus relaciones de pareja y las implicancias que estos significados tienen en sus interacciones sociales. Los objetivos específicos son dos:

Identificar los distintos significados que tienen los y las estudiantes universitarios sobre sus relaciones de pareja Determinar las implicancias, de los distintos significados que los y las estudiantes universitarios tienen sobre sus relaciones de pareja, en sus interacciones sociales.

\section{MATERIAL Y MÉTODOS}

Es una investigación cualitativa. El universo está constituido por 6433 estudiantes matriculados en el año académico 2011 en la Universidad Nacional Jorge Basadre Grohmann de Tacna. La muestra está dada por 20 estudiantes, hombres y mujeres en los que se estudió la variable: Significados sobre las relaciones de pareja y sus implicancias en las interacciones sociales.

Se utilizó la entrevista a profundidad y grupo focal.

\section{RESULTADOS}

\section{Lo que significa una relación de pareja.}

Los hombres, aclaran que no todas las relaciones amorosas pueden ser consideradas una relación de pareja. Probablemente aludan a las relaciones denominadas "choque y fuga" que los jóvenes de hoy suelen considerar. La variable que consideran es el tiempo que dura una relación: "Hay relaciones que duran una semana o dos o un mes esas no son relaciones de enamorados". Juan

La definición de relación de pareja incluye: inversión de tiempo, compartir cariño y amistad.

Las jóvenes tienen una definición más amplia que los varones respecto a lo que significa tener una relación de pareja: amar, sentirse bien, compartir, y confianza:

"Es compartir alegrias y tristezas y tener confianza". Cecy
Los hombres, en la relación de pareja, encuentran bienestar que satisface sus necesidades de compañía y de conocerse a sí mismos:

"Me gustaba salir, hablar de cosas, sentirme bien. Ella era alegre, bromista, es lo que más me gustó". Gian

\section{Cómo construir el vínculo amoroso.}

El pensar como unidad fortalece el vínculo amoroso, gana fuerza, se establecen ciertas condiciones que le dan estructura a la relación:

"No ser infiel, tener tiempo para ella, que respeten tus espacios (por ejemplo el tiempo de los estudios). Las reglas no se dicen, se sobreentienden. Ya se sabe, cuando hay problemas se sabe que se deben respetar las reglas" Gian

Una relación de pareja está marcada por la inversión que ambos hacen en un vínculo amoroso, impulsados por el deseo de desarrollar un proyecto conjunto, cuando se reconocen como enamorados. Este reconocimiento es social y personal y supone un compromiso en el momento en que perciben que es una relación con cierta estabilidad. En esta posición, tanto los hombres como las mujeres consideran que deben combinar organización de su tiempo, momentos de diversión y seguridad como miembros de la pareja:

"Si no hay confianza no funciona. Cuando hay celos la relación no es buena". Mary

Las mujeres consideran que es posible mantener una relación de pareja a distancia, consideran que las nuevas tecnologías de la información son sus aliadas y les permite "estar juntos". Para los varones, la distancia no es una barrera que se pueda derribar para mantener una relación. Ellas sobrevaloran el elemento romanticismo asociado a la comunicación y la confianza en la relación.

\section{El fortalecimiento de la relación de pareja.}

Para las mujeres, el trato autoritario de su pareja no es aceptable ello significaría no respetar el espacio del otro. Esto no suele ser percibido antes del inicio de la relación sino durante el desarrollo de la misma y puede denotar engaño y desencanto que conduce al fin de la relación, a pesar de que se pueda reconocer otros rasgos positivos de la pareja:

"Es inteligente, carismático, hasta que conoci su otra cara. Su mamá lo trata como él me trataba a mí. Su mamá es autoritaria, su papá indiferente, su hermana muy engreída". Rosa

Son rasgos deseables en la pareja: la bondad, amabilidad, ser detallista, comprensivo, respetuoso. Son rasgos no aceptables, según las mujeres, ser celoso porque ello se entiende como una falta de confianza:

"Un día me acompañó a comprar un polo y no le gustó porque estaba "muy escotada" según él, se enojó y tiró los libros al suelo. Esa actitud no me gustó, me hizo sentir mal, la gente me quedó mirando, sentí ganas de llorar, me senti pésimo, me senti mal, no habia hecho nada malo" Julia 
Las mujeres aprecian los regalos de sus parejas, como muestras de amor. Parece haber la percepción de que para que la relación amorosa dure en el tiempo y exista la posibilidad de un proyecto a futuro, la pareja necesita reforzar la seguridad con manifestaciones afectivas y materiales. Este reforzamiento tiene que ver con la comunicación de la pareja, la fidelidad y entrega de objetos demostrativos del amor:

"Mi segundo enamorado: muy detallista, comprensivo, amable. Me respeta y cuida". July

Los hombres creen que las mujeres le dan mucha importancia a ser detallista y eso puede incomodarlos, porque piensan que ellas prefieren los regalos materiales:

"No vale tanto lo material, pienso yo y se lo decia a ella, pero a las mujeres les gustan estos detalles, yo creo que también podria ser un regalo un poema". Carlos

Cuando las expectativas que cada miembro de la pareja no son satisfechas, surgen los conflictos porque no se cumplieron con las condiciones y roles que ambos establecieron: invertir tiempo, mostrar pruebas materiales de amor ("detalles"), tener buena comunicación, respetar los espacios de desenvolvimiento de cada uno:

"Mi relación duró unos meses pero terminó por falta de confianza (celos). Llegó a algo serio. Algo serio es que llegas a conocerla bien, que hay más cariño, respeto, el cariño aumenta. Gian

No parece importante la intervención de la familia en el fin de las relaciones de pareja. Ellos y ellas reconocen que sus padres pueden opinar, pero parecen ignorar sus opiniones porque podrían considerarlas exageradas.

Una forma de proteger su relación de pareja, es manteniéndola en el desconocimiento de sus padres. Esto deja traslucir que en la mayoría de los casos los y las jóvenes consideran que sus padres no podrían estar de acuerdo con que ellos(as) tengan una relación:

"Yo termine mi relación, por una parte por mi familia, pero luego volvimos y estábamos en secreto solo lo sabian mi hermanay su hermana. Naby

Los hermanos y hermanas también están presentes en la relación de pareja, como espectadores, ejemplos de relaciones de pareja, como consejeros(as), probablemente su presencia sea mayor que la de los padres en este tema:

"Mis padres no hablan de enamorados (pareja). En 4to. Tuve un enamorado, se enteró mi hermano y me hablaba y aconsejaba, decia que ellos sólo querian acostarse y ahora en la Ume dice que debo de estar segura de lo que haga, me sobreprotege, soy la única mujer". Cecy

Si bien las opiniones de los padres no son importantes para finalizar una relación de pareja, los jóvenes escuchan de ellos sus mensajes respecto a sus temores frente a las consecuencias de una relación amorosa, estas preocupaciones están relacionadas con la sexualidad:

"Si fui presionada por mi papá y mamá, no querian que tenga enamorado, mi papá tenía miedo queme embarace quería que estudie. Pensaba en lo que diría la gente si estaba con uno yotro chico". Julia

Los hombres reconocen a la madre como su aliada para el desarrollo de su relación de pareja. La comparan con el padre, y ella resulta ser más comprensiva, tolerante e interesada.

Además del temor al embarazo, los padres tienen el temor de que sus hijos al ingresar en una relación de pareja, descuiden sus estudios. Los hombres creen que esta idea es exagerada, y que más bien, ellos son capaces de cambiar ciertos hábitos que consideran no muy favorables en su vida personal: dejan de beber, aprenden a despegarse de los amigos para pensar en la pareja, estudian con más ahínco para terminar rápido los estudios y poder plasmar su proyecto de una vida junto a su pareja:

"Los padres... ellos piensa que bajas notas por tener enamorada porque haces más caso a tu relación, a tu enamorada, que a tus estudios". Juan

Los amigos también están presentes, como espectadores que en ocasiones hacen escuchar su voz:

"Mis amigos me felicitaron por tener enamorada". Juan

Una causa importante del rompimiento de una relación de pareja puede ser la violencia. Aquí pueden estar presentes factores como la infidelidad, la falta de sinceridad y la pérdida de reconocimiento social de la pareja:

...peleábamos a diario, me seguía, si mis amigos me hablaban o saludaban se enojaba, gritaba, golpeaba las paredes. Peleábamos lejos de la U, para los demás éramos la pareja perfecta. En una pelea llegó a "lastimarme" pero pidió perdón, a la semana otra vez. Me agarraba de los brazos y me apretaba tan fuerte que me dejaba "rojo" y me decia "sólo te quiero cuidar". Era muy absorbente, a toda costa quería estar conmigo. Mary

El "duelo" por el rompimiento de una relación de pareja, en la experiencia de los hombres, es distinto según quién es el causante del rompimiento. El sufrimiento es mayor para el hombre si es él quien terminó la relación, porque está haciendo sufrir a una persona que lo amó o lo ama. Si la que termina la relación es la mujer, él duelo dura menos tiempo.

\section{Proyecto de pareja. La necesidad de llegar al matrimonio.}

Una característica en la definición de relación de pareja es el sentimiento de compromiso basado en sentimientos de apego y el deseo de mantener una relación duradera. Es en estas circunstancias que surge en la pareja la idea de matrimonio. Entre las jóvenes está más presente la idea de matrimonio.

Ellas han desarrollado la idea de la unión futura y han pensado inclusive en que parte de esta unión será tener hijos:

"Ahora no, cuando termine la carrera, para poder formar una familia, que él también estudie y tener trabajo. Me gustarian 2 hijos, dependerá de la economia, del trabajo". Cecy 
En las mujeres la idea de casarse ha nacido en el seno de su hogar ideas como" que las hijas salen de la casa casadas" y la idea del matrimonio religioso. Se puede percibir el romanticismo en sus comentarios:

"Por mis papás, ellos quieren que todos sus hijos estén casados. Mis hermanos ya se casaron ". Rosa

Los hombres se muestran más precavidos al tocar el tema del matrimonio. Se esfuerzan por aclarar que sólo en alguna de sus relaciones amorosas existió esa idea de matrimonio.

Tanto mujeres como hombres establecen ciertas condiciones para llegar al matrimonio: terminar la carrera, tener trabajo y tener cierta edad. Las mujeres tienen menos expectativas que los hombres respecto a cuánto esperar para un matrimonio (terminar la carrera y trabajar). Los hombres consideran que este trabajo debe ser estable, tener dinero, tener propiedades, haber continuado estudios luego de acabar su carrera actual, y en esta situación su edad para el matrimonio es mayor que el que consideran las mujeres.

Las mujeres y los hombres identifican dos características en el matrimonio: la responsabilidad de tener hijos (consideran que si el matrimonio fracasara los perjudicados serían los hijos) y el temor a la posibilidad de un rompimiento matrimonial:

"Sí, no es un juego, si tienes hijos los perjudicados son ellos. Mary

"Si, para siempre". Cecy

Los hombres consideran que lo que podría llevar a un rompimiento del matrimonio es la infidelidad y la falta de ingresos económicos:

"Por falta de tiempo, trabajar todo el dia y no estar con la pareja. Si puede haber separación por falta de ingresos económicos, el amor no solo se acaba por infidelidad".Juan

\section{Ejercicio de la sexualidad en la relación de pareja. La intimidad con la pareja antes del matrimonio.}

Entre las mujeres encontramos posiciones en contra y a favor de las relaciones prematrimoniales. No sólo las desaprueban sino que el sólo hecho de la proposición de la intimidad es considerado como una falta de respeto. Además, está presente la idea de tener una sola pareja sexual durante toda su vida, obviamente con la persona con la que contraerían matrimonio. Entre las mujeres que están a favor de las relaciones sexuales prematrimoniales (y que ya se iniciaron sexualmente), la intimidad está sujeta a sentimientos de seguridad respecto a la pareja y su preparación emocional para aceptar lo mismo:

\footnotetext{
"Mi pareja me pedia tener relaciones sexuales, yo no estaba segura, supo esperar, lo pensé y lo hice cuando estuve segura". Julia
}

\footnotetext{
"Antes de tener relaciones sexuales debo conocerlo, si lo hago será con quien esté toda la vida, puede ser antes o después". Cecy
}

Entre los hombres las opiniones sobre las relaciones prematrimoniales están influenciadas por cuestiones religiosas y la crítica de la sociedad, pero no niegan ni afirman su disposición personal respecto a si ellos tienen o tendrían relaciones prematrimoniales:

"Mi religión tiene que ver con mi decisión de seguir virgen hasta mi matrimonio. Si la relación hubiera durado más tiempo hubiera llegado al sexo". Naby

\section{"La sociedad no puede criticarnos por tener relaciones coitales antes del matrimonio”. Gian}

En general, los y las jóvenes dicen que no se habla de tener sexo en la relación, al menos no abiertamente o como una condición para continuar la relación:

"No se toca el tema del sexo. No se habla de tener o no relaciones coitales". Naby

Al hablar de relaciones sexuales, los y las jóvenes lo asocian al matrimonio. Los hombres muestran que tienen un gran temor al matrimonio. Ellos proponen que una relación de pareja debe tener facetas: del enamoramiento, de la convivencia y del matrimonio. La etapa del enamoramiento básicamente sería para fortalecer la amistad y el reconocimiento de sus habilidades personales para estar en la relación, y esta etapa podrían o no haber relaciones sexuales. En la etapa de convivencia estarían presentes las relaciones sexuales, se tendrían hijos; y la pareja se conocería en su práctica de valores, fundamentalmente el de la responsabilidad y el de la solidaridad:

"Yo estoy de acuerdo. Vivir juntos antes de casarse es bueno para saber si nos llevamos bien porque eso no se puede saber en el enamoramiento". Naby

Los hombres tienen también la clara idea de que el período de convivencia debe ser mayor al período del enamoramiento.

Los hombres diferencian el significado de noviazgo al de la convivencia. Dicen que en el noviazgo existe un compromiso de casamiento, en la convivencia no, pues está claro que es una prueba que se puede o no pasar. Sólo si el balance es positivo habría matrimonio, sino cada uno, tomaría rumbos distintos sin compromisos:

"Hay diferencia entre noviazgo y convivencia. Noviazgo estás comprometido a que si te vas a casar: En la convivencia no hay ese compromiso". Gian

\section{DISCUSIÓN}

\section{Significado de la relación de pareja.}

Para Leñero, la formación de la "pareja humana" hombre-mujer responde a una realidad bisexual del género humano, necesaria para "producir y reproducir su existencia [...] Juntos forman a la sociedad, el varón se define por la mujer y viceversa" (Leñero; 1983,195). Los y las jóvenes de este estudio la definen como un vínculo amoroso basado en el compartir sentimientos y compañia. Para Stendhal (citado por Alberoni, 1992), el enamoramiento es un proceso unilateral por el cual embellecemos (cristalizamos) las cualidades de la persona amada" (Alberoni; 1992, 64). 
Los significados que los y las jóvenes tienen sobre las relaciones de pareja no son estáticos en el tiempo, estos van cambiando dependiendo del tiempo y espacios históricos en el que estos viven. Esto explica que entre los estudiantes entrevistados hombres haya surgido la propuesta de la convivencia antes de un compromiso para el matrimonio como una forma de evitar las consecuencias de un fracaso matrimonial, la cual identifican como una etapa de la relación de pareja que permite la práctica de relaciones coitales. Sin embargo, en las mujeres encontramos que sigue prevaleciendo un modelo tradicional de pareja: enamoramiento, noviazgo y matrimonio.

Lazo López, en su trabajo Imaginario moderno sobre las relaciones de pareja de los jóvenes, señala que es posible identificar dos modelos sobre las relaciones de pareja: el modelo tradicional y el modelo liberador. El primero tiene que ver con el amor romántico, la lealtad, fidelidad, compromiso y noviazgo. El segundo representa aquellas relaciones casuales, temporales y hedonistas. Para este autor, el segundo modelo está altamente influenciado por la globalización y la influencia de la modernidad occidental. Pero los y las jóvenes de este estudio, a pesar de la globalización y la influencia de una modernidad occidental y norteamericana, no han modificado sustancialmente sus imaginarios respecto a la relación de pareja. No se percibe que ellos establezcan relaciones de pareja para tener actividad sexual fundamentalmente (lo cual es típico en el modelo liberador mencionado por Lazo López). Sin embargo, tampoco, en su elección de pareja está presente el idealismo que haga que relacionen su enamoramiento como un hecho predestinado o que lo vivan ilusos pues reflexionan sobre su relación en el sentido de que saben que la formación de una pareja es una experiencia humana que se vive con un grado importante de incertidumbre, y que por lo tanto si se quiere tener éxito en esto se tiene que cumplir con ciertas pautas.

Holmes (2000) encontró que las personas que tenían una idea basada en "el destino" respecto a encontrar el ideal de pareja, tendía a presentar más quiebres de la relación cuando vivían experiencias de insatisfacción, en comparación con quienes sustentaron teorías de crecimiento que enfatizaban la capacidad de las relaciones de desarrollarse en el tiempo. Es decir, que es positivo que los y las jóvenes del estudio sepan que sus interacciones en la relación de pareja puedan hacer que esta llegue a un final, pues estarán en mejor posición para aceptar este quiebre en su vida, y podrán continuar con la misma superando en el breve tiempo las carencias afectivas causadas por la ruptura.

\section{Proyección de la pareja.}

La formación de una pareja es una experiencia humana que se vive con incertidumbre. Holmes (2000) encuentra que en la relación de pareja operan mecanismos de interpretación, compensación, ilusión, reducción de la disonancia, evaluación, los cuales parecen mostrar una mayor capacidad predictiva y explicativa de la estabilidad y el grado de satisfacción en la pareja, en comparación con aspectos conductuales u "observables". Esto sería consistente con lo que encontramos en la presente investigación, respecto a que los jóvenes y las jóvenes consideran que al estar en una relación de pareja ellos son capaces de poner esta relación por encima de su familia y de sus amigos(as), pues con la pareja se sienten bien y sienten la necesidad de interaccionar con ella, de manera que si esta necesidad crece puede surgir la idea de un proyecto de pareja, que involucre su futuro.

Pero ¿qué lleva a la pareja a pensar en prolongar en el tiempo la relación? Los jóvenes y las jóvenes de este estudio señalan que "tener detalles con la pareja" y "tener una buena comunicación" son factores determinantes en una relación.

Como "detallista", se entiende como hacer regalos materiales e inmateriales y recordar aniversarios. Confirmando esta situación, encontramos a Kelley (1979), que utilizando su modelo de la interdependencia considera los efectos de la interacción. El explica que en una relación se dan procesos de recompensa y costos, observando que no sólo se involucra una producción individual de estos costos y beneficios, sino también una generación conjunta de éstos. Así pues, los costos y beneficios de la relación de pareja están dados por recibir regalos materiales o inmateriales como pruebas de amor y necesidad de la presencia de la pareja los cual es interpretado como estar enamorado o mostrar predisposiciones personales en conductas observables para mantener la relación. Ello hace surgir la idea de compromiso y el estar comprometido(a) puede servir para valorar más la relación, y la relación es menos vulnerable al rompimiento.

Llama la atención que los jóvenes del estudio no hablen de lo que ellos dan sino de lo que ellos reciben. Probablemente esto se deba a que los entrevistados sean muy jóvenes y hayan tenido pocas experiencias de relaciones de pareja y/o que en la mayoría de los casos no han sido muy duraderas ( 5 meses en la mayoría de los casos), y en este sentido la relación terminó o no ha evolucionado y sus miembros no han encontrado la satisfacción en su interacción como pareja.

La formalización de una relación de pareja se da si hay satisfacción entre los integrantes. Esta satisfacción es una apreciación subjetiva que cada individuo hace del bienestar, calidad y valor de las diferentes facetas de su pareja y de su interacción en ella. Cada integrante de la pareja está seguro de que ambos comparten un sentimiento que les da bienestar y desean prolongar este bienestar en el tiempo, esto hace que piensen en el matrimonio. Suponen que en el matrimonio su relación encontrará estabilidad y saben que tendrán que enfrentar retos, los cuales estarían dispuestos a asumir.

El psicólogo norteamericano Hendrix (1997, citado por Pinto Tapia), utiliza el término "imago" para referirse a la construcción ideal que hacemos de nuestras expectativas amorosas, a partir de los esquemas afectivos y cognitivos aprendidos en nuestra familia, por ello esperamos la satisfacción de nuestras expectativas afectivas, aquellas que nos dieron satisfacción y las que 
nos faltaron. Esto coincide con el hecho de los y las jóvenes que participaron en esta investigación, a pesar de que afirman que sus padres no interfieren en sus relaciones de pareja, ello no es muy cierto o no tratarian de mantener en secreto sus relaciones.

\section{Ejercicio de la sexualidad en la relación de pareja.}

Las mujeres y hombres de este estudio jerarquizan los lazos del vínculo en la relación de pareja. Señalan que la relación de pareja se fortalece con la amistad, la comunicación, el cariño, el apoyo emocional, pero excluyen como un fuerte lazo de unión la actividad sexual.

Además, está presente la idea de conservar la virginidad hasta el matrimonio tanto en hombres como en mujeres. Parece haber una resistencia de los y las jóvenes de aceptar abiertamente que la vida sexual tiene mucha importancia en una relación de pareja, que en el amor humano están presentes el componente físico, el psicológico y el espiritual. No se puede negar que el amor en una relación de pareja nace de la atracción física, por eso las relaciones entre amor y actividad sexual son indesligables.

\section{CONCLUSIONES}

1. En la relación de pareja concurren procesos cognitivos que se concretan en el espacio psicológico y el espacio físico. Estos procesos tienen que ver con el reconocimiento de la identidad personal, el descubrimiento de la necesidad de compartir sentimientos y espacios de la vida personal con otra persona interesada también en hacerlo.

2. Los y las jóvenes de la actualidad basan sus relaciones de pareja en las interacciones de uno y otro miembro en la relación. Es decir, de la forma en que ambos asumen los costos y beneficios de la relación: compartir sentimiento, mostrar conducta s y actitudes observables que demuestren interés por fortalecer el vínculo amoroso.

3. La infidelidad y la falta de conductas y actitudes observables que demuestren el interés por fortalecer el vínculo amoroso, así como la falta de una adecuada comunicación, son las causas del fin de la relación entre los jóvenes de la actualidad. La comunicación

satisfactoria es considerada por los y las jóvenes universitarias como imprescindible para manejar los conflictos al interior de la pareja y evitar el rompimiento de la relación.

4. Las relaciones sexuales son un componente de la relación de pareja en los jóvenes, aún y cuando estas no se den en todas sus relaciones la idea del contacto físico intimo está siempre presente en sus imaginarios.
5. Los hombres consideran que una relación de pareja debe tener tres fases: enamoramiento, convivencia y matrimonio. La última condicionada a la superación exitosa de la fase de convivencia. Esta idea no está presente en las mujeres, lo que podría significar que las mujeres no han superado el modelo de relación de pareja tradicional y que los hombres se inclinan por aceptar un modelo de relación de pareja liberal.

6. En las mujeres la idea del matrimonio ha nacido en el seno de su hogar, asociado a la religión y al mito de la virginidad.

7. Las jóvenes universitarias han enfrentado situaciones de violencia de parte de los hombres en su relación de pareja. Esta violencia es demostrada a través de conductas de impotencia, autoritaria, celos y desconfianza

\section{REFERENCIAS BIBLIOGRÁFICAS}

Alberoni, Francesco, (1992) El vuelo nupcial, Editorial. Gedisa, Barcelona, España.

Driceida Pérez R. (2000). Significados Sociales En Las Relaciones De Pareja.

Fernández R., Lourdes (2006) Personalidad y relaciones de pareja. La Habana: Editorial Ciencias Médicas. Correo Cientifico Médico de Holguín 2009; 13(2). Al 25/3/2012. Disponible en:

http://www.cocmed.sld.cu/no132/no132rev2.htm

Flores C, et al (2004). Jóvenes universitarias que legitiman la violencia en sus relaciones de pololeo. Tesis para optar al Título de Asistente Social, Licenciado en Desarrollo Familiar y Socia. Universidad Católica de Temuco- Chile. Facultad de Artes, Humanidades y Ciencias Sociales. Escuela de Trabajo Social

Garduño Duarte, Aurora. La significación cognoscitiva y las causas que determinan el noviazgo, matrimonio y unión libre en un grupo de universitarios de 20 a 30 años de edad que viven en la ciudad de México, Tesis de la Facultad de Psicología, UNAM, 2002.

Gergen, K. (1973). Social Psychology as History. Journal of Personality and Social. Psychology, 26(2), 309-320.

Gergen, K. (1999). An invitation to Social Construction. Sage Publications, London

González A, Electra et al. Percepciones y experiencias del inicio sexual desde una perspectiva de género, en adolescentes consultantes en un centro universitario de salud sexual y reproductiva. Rev chil. obstet. ginecol. [online]. 2010, vol.75, n.2 [citado 2012-03-26], pp. 8490. Disponible en:

$<\mathrm{http}$ ://www.scielo.cl/scielo.php?script=sci_arttext\&p id $=$ S0717 
Holmes, J. (2000). Social relationships: the nature and function of relational schemas. European Journal of social psychology, 30, 447-495.

Kelley, H. (1979). Personal relationships: Their structure and processes. Hillsdale, NJ: Earlbaum. Cap. 2 (pp.13-55).

Lemaire G. (1995), Jean La pareja humana: su vida, su muerte. La estructuración de la pareja humana FCE, México.

Leñero, O Luis. (1983). El fenómeno familiar en México. IMES, México.

Lizana Muñoz, Verónica Alejandra. Representaciones sociales sobre feminidad de los/las estudiantes de pedagogía, en los contextos de formación docente inicial. Estud. pedagóg. [online]. 2008, vol.34, n.2 [citado 2012-03-26], pp. 115-136. Disponible en: $<\mathrm{http}: / /$ www.scielo.cl/scielo.php?script=sci_arttext\&pi $\mathrm{d}=\mathrm{S} 0718$ -

Moraleda, Mariano, (1997) Psicología del desarrollo, Editorial Boixareu universitaria, España 1992.

Murray, S. \& Holmes, J. Leap of faith? Positive Illusions in romantic relationships. Personality and Social psychology Bulletin, 23, 586-604.
Romo Martínez, José Matías (2008): Estudiantes universitarios y sus relaciones de pareja. Revista Mexicana de investigación educativa, julio-septiembre, año/ vol. 13, número 038. México.

Sánchez A., Rozzana y Díaz Loving, Rolando. (2003). Patrones y estilos de comunicación de la pareja: Diseño de un inventario. Anales de psicología, 2003, vol. 19, $\mathrm{n}^{\circ}$ 2 (diciembre), 257-277.

\section{WEBGRAFÍA}

Lazo López Luis Miguel. Imaginario moderno sobre las relaciones de pareja de los jóvenes $<$ !--[if !supportFootnotes]-- $>[1]<!--[$ endif]-- $>$

\section{Correspondencia:}

Carmen Luisa Linares Torres

Ciudad Universitaria Fundo "Los Granados"

Av. Miraflores s/n Tacna Perú clinares40@hotmail.com

Gema Natividad Sologuren García Ciudad Universitaria Fundo "Los Granados"

Av. Miraflores s/n Tacna Perú 